\title{
A Medicina Interna na Ortopedia: Relato de uma Experiência
}

\section{Internal Medicine in Orthopedics: An Experience Report}

Isabel Silva ${ }^{1,2}$, Maria João Silva'1, Alexandre Carvalho',2, Paulo Cibrão Coutinho³, Rui Duarte ${ }^{3}$, Paulo Fragoso ${ }^{4}$, Manuel Vieira da Silva ${ }^{3}$, António Oliveira e Silva ${ }^{1}$

Autor Correspondente:

Isabel Silva [imas.silva@gmail.com]

\section{RESUMO}

INTRODUÇÃO: O envelhecimento crescente da população e a necessária gestão de doentes cada vez mais complexos numa sociedade onde se esperam melhores resultados do ponto de vista custo-efetividade tem exigido aos serviços de saúde uma visão necessariamente mais multidisciplinar das especialidades.

MÉTODOS: Neste artigo apresenta-se uma breve casuística de três meses da articulação direta diária entre ortopedistas e internistas, assentes numa prestação de cuidados integrados aos doentes internados por causas ortopédicas mas com necessidade de gestão de comorbilidades e/ou intercorrências significativas. Aborda-se ainda o protocolo das fraturas proximais do fémur criado por uma equipa de trabalho interdisciplinar no sentido de prestar melhores cuidados a uma situação ortopédica associada a considerável morbimortalidade.

RESULTADOS: Na maioria dos casos os doentes estavam internados em contexto de patologia traumática e foram submetidos a tratamento cirúrgico. Destacaram-se a elevada faixa etária, a diversidade de comorbilidades, o número importante de infeções nosocomiais e a necessidade de gestão médica multidisciplinar. A maioria dos doentes exigiu acompanhamento regular.

CONCLUSÃO: A perceção de doentes com elevado número de comorbilidades e outras entidades nosológicas a par do problema ortopédico salienta a necessidade de um acompanhamento mais holístico, onde certamente a Medicina Interna pode exercer um papel fundamental.

PALAVRAS-CHAVE: Comportamento Cooperativo; Equipa de Assistência ao Doente; Fraturas do Fémur; Medicina Interna; Ortopedia

\section{ABSTRACT}

INTRODUCTION: The increasing aging population and the necessary management of increasingly complex patients with multiple comorbidities in a society where the results are measured in terms of cost-effectiveness, health services must focus on multidisciplinary team work. 
METHODS: This article presents a brief sample of three months of daily direct work between orthopedic surgeons and internists, based on a provision of integrated care to hospitalized patients for orthopedic causes but who need management of comorbidities or significant pathological conditions. The authors also address the proximal fractures of femur protocol created by an interdisciplinary working team sense to provide better care to an orthopedic condition associated with considerable morbidity and mortality.

RESULTS: In most cases patients were hospitalized in traumatic context and underwent surgery. The authors highlight the high age, the diversity of comorbidities, the relevant number of nosocomial infections and the need for multidisciplinary medical management. Most patients required regular monitoring.

CONCLUSION: The perception of patients with high number of comorbidities and other nosological entities alongside the orthopedic problem, stresses the need for a more holistic care, where certainly Internal Medicine can play a key role.

KEYWORDS: Cooperative Behavior; Internal Medicine; Hip Fractures; Orthopedics; Patient Care Team

\section{INTRODUÇÃO}

"All special branches of medicine, orthopedic surgery included, must preserve their proper relations with internal medicine and that patients must not be treated as collections of unrelated special organs, but as groups which are combined always into single physiological entities."

(Boston Medical and Surgical Journal, 1913). ${ }^{1}$

A Medicina Interna é uma especialidade de carácter integrador, que se dedica à prevenção, diagnóstico e tratamento não cirúrgico de uma grande variedade de patologias que afetam um ou mais órgãos ou sistemas. A conjugação entre a abordagem global das necessidades do doente, o diagnóstico e tratamento de uma variedade de patologias e a mediação de intervenções por outras especialidades, torna o Internista um elemento essencial no acompanhamento do doente em todo o seu percurso hospitalar. Um estudo liderado por Pedro Pita Barros (2007) da Faculdade de Economia da Universidade Nova de Lisboa mostrou que o aumento do número de clínicos de Medicina Interna nos hospitais poderia representar uma poupança de 32 milhões de euros, concluindo que "a sua intervenção contribui para a prestação de cuidados mais eficientes e maior controlo de custos". ${ }^{2} \mathrm{O}$ crescente consumo de cuidados por parte das populações cada vez mais idosas tem determinado uma absorção progressiva de recursos no sistema de saúde; todavia, as questões da eficiência têm dominado as políticas de saúde nos países mais avançados. A divisão do trabalho hospitalar baseada nas especialidades médicas e cirúrgicas parece hoje querer seguir um percurso diferente. Uma visão mais integrada do doente e da doença, num trabaIho mais multidisciplinar, cria novas responsabilidades à Medicina Interna, nomeadamente na gestão do doente internado, mas certamente contribuirá para melhores resultados do ponto de vista custo-efetividade. ${ }^{3}$

O Hospital de Braga tem uma área de influência global de cerca de 1.2 milhões de habitantes e uma área de influência direta de 300.000 habitantes. Conta com 705 camas de internamento, assumindo os Serviços de Me- dicina Interna e de Ortopedia uma percentagem importante da sua ocupação (95 e 90 camas respetivamente). Tendo em conta o envelhecimento crescente da nossa população, a necessária gestão de doentes cada vez mais complexos, e o elevado número de pedidos de colaboração de consulta interna habitualmente feitos pela Ortopedia à Medicina Interna, foi decidido em maio de 2014 destacar dois elementos do Serviço de Medicina Interna para exercer apoio a tempo inteiro no Serviço de Ortopedia.

Os autores apresentam uma breve casuística relativa à experiência de três meses de internistas em funções a tempo inteiro no Serviço de Ortopedia, procurando perceber o contexto dos doentes internados e o tipo de articulação ativa que se pode estabelecer entre ambas as especialidades, com vista à melhoria dos cuidados aos doentes, como se exemplifica pelo protocolo das fraturas proximais do fémur (FPF) criado.

\section{METODOLOGIA}

Estudo retrospetivo relativo ao primeiro trimestre de 2015. Doentes internados no Serviço de Ortopedia do Hospital de Braga em que foi solicitada observação e colaboração por Medicina Interna (pedido escrito no sistema informático, com registo no processo clínico do doente). Breve enquadramento do protocolo FPF. O protocolo FPF foi desenvolvido em parceria entre a Ortopedia, a Medicina Interna e a Anestesiologia e tem como objetivo prestar uma especial atenção e cuidados mais rápidos e mais completos numa intercorrência que se associa habitualmente a elevada morbimortalidade, devendo ser assegurado o tratamento cirúrgico até às 48 horas após admissão hospitalar. Após admissão no Serviço de Urgência (SU) e feito o diagnóstico de fratura proximal do fémur, o doente deverá ser internado na enfermaria no período de duas horas com história clínica sumária e prontamente referenciado pelo Ortopedista para Medicina Interna e pedida a avaliação pré-anestésica por Anestesiologia. A avaliação por Medicina Interna 
deve ser feita nas primeiras 12 a 24 horas após internamento, para todos os doentes referenciados. A avaliação por Anestesiologia deve ser feita também nas primeiras 12 a 24 horas após internamento, preferencialmente após avaliação e orientação por Medicina Interna. Os doentes que integram o protocolo FPF têm prioridade de avaliação por Medicina Interna sobre os outros doentes ortopédicos em que também é solicitado o apoio desta especialidade, exceto quando se tratam de situações de carácter urgente. Este protocolo estabelece ainda linhas orientadoras relativamente aos exames complementares de diagnóstico "padrão" a solicitar, assim como a medidas de tromboprofilaxia, de profilaxia de infeção da ferida cirúrgica, de analgesia e de reabilitação.

\section{RESULTADOS}

Foram observados 88 doentes, e a média de idades foi de 79 anos, tendo o doente mais novo 45 anos e o mais velho 95 anos. Nos 88 doentes observados, o sexo feminino $(n=55)$ prevaleceu sobre o sexo masculino ( $n=33)$ os resultados em percentagem estão expressos na Fig. 1. Dos doentes, 62\% eram previamente autónomos, 29\% parcialmente dependentes e apenas $8 \%$ totalmente dependentes.

Os motivos de internamento em Ortopedia dos doentes analisados foram, na sua maioria, devidos a patologia traumática, sendo que 60 dos casos correspondiam a doentes com fratura proximal do fémur (assumindo uma grande representação as fraturas subtrocantéricas e as do colo). Os dados encontram-se discriminados na Fig. 2. A grande maioria dos doentes em questão ( $n=78)$ foi submetida a tratamento cirúrgico, tendo dez sido alvo de tratamento conservador (três casos de espondilodiscite e sete casos de patologia traumática que não reuniram condições cirúrgicas). Dos 78 doentes operados, 73 correspondiam a situações de patologia traumática aguda (fraturas) e apenas cinco correspondiam a casos de cirurgia programada (um caso de prótese total da anca (PTA), um caso de prótese total do joelho (PTJ), um caso de artroplastia da anca e dois casos de artrite séptica do joelho por PTJ infetada).

Uma percentagem importante dos doentes $(n=31$, 42,5\%) foi intervencionada nas primeiras 48 horas após a intercorrência traumática, considerado na literatura o timing ideal para o efeito por se associar a menor morbimortalidade. Ainda assim, têm sido feitos esforços cada vez maiores para aumentar esta cobertura, mesmo em período de fim de semana, sendo este um dos principais objetivos propostos no algoritmo de abordagem do doente com fraturas proximais do fémur (Fig. 3). Dados disponibilizados pelo Serviço Nacional de Saúde (SNS) e Administração Central do Sistema de Saúde (ACSS), utili-

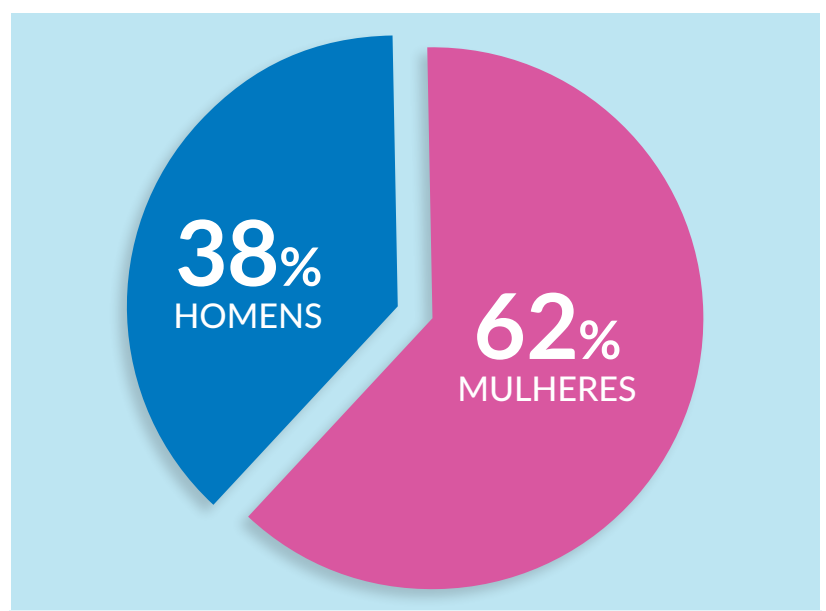

FIGURA 1. Distribuição dos doentes observados por Medicina Interna, por sexo (em percentagem, para um $\mathrm{n}=88$ ).

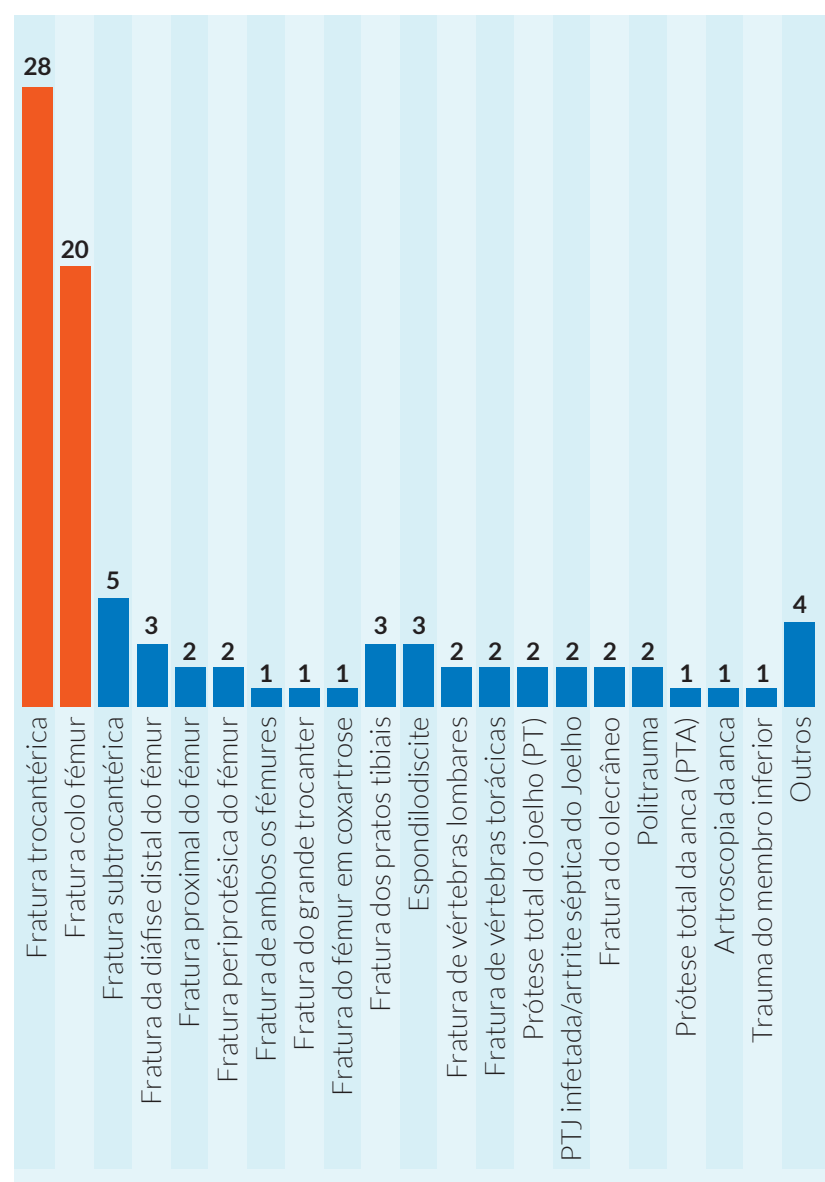

FIGURA 2. Motivo de internamento ortopédico nos doentes em que foi solicitado o apoio de Medicina Interna (valores individuais).

zando como indicador a percentagem de fraturas da anca com cirurgia realizada nas primeiras 48 h, mostram que no Hospital de Braga essa cobertura foi de 42,07\% em junho de 2014 (data de início do protocolo FPF) e passou para 69,23\% em junho de 2015, o que traduz uma vez mais o impacto das medidas instituídas e permite colocar o Hospital num melhor grau de desempenho (Fig. 4).

Foi necessário adiar o timing da cirurgia em nove doentes. Relativamente aos motivos pelos quais houve necessidade desse adiamento verificou-se o seguinte: três ca- 

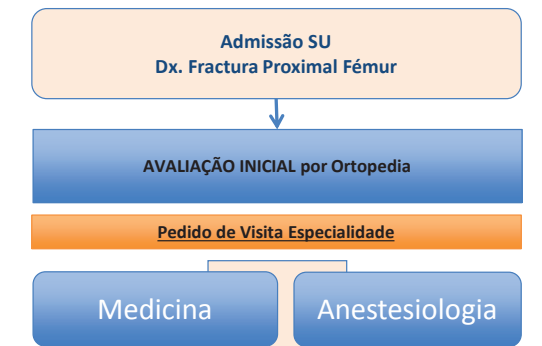

Medicina

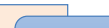

Anestesiologia

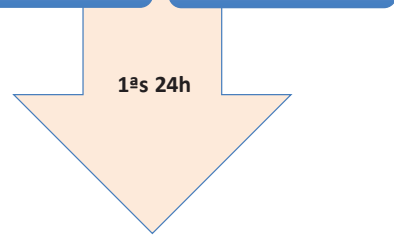

20
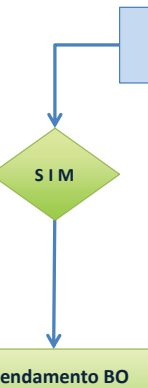

Agendamento BO

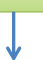

Cirurgia até às 48

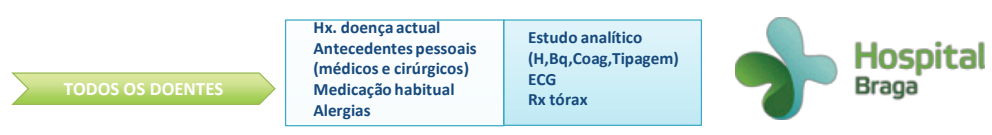

FIGURA 3. Algoritmo de abordagem do doente com fraturas proximais do fémur, Hospital de Braga (elaborado em maio de 2014).

sos por febre, um caso por taquicardia de novo, um caso por hipercaliemia, um caso por síndrome confusional aguda, um caso por infeção respiratória, um caso por infeção dos tecidos moles pélvicos e um caso por ausência de jejum. Nestas situações, o adiamento cirúrgico foi da iniciativa do Anestesista, do Internista ou do Ortopedista. Nos restantes casos de doentes operados o adiamento cirúrgico foi devido a falta de agendamento.

Tendo em conta o total de 88 doentes observados, 46 foram por pedidos no âmbito do protocolo FPF e 42 por outros motivos, como se expressa na Fig. 5. Estes últimos corresponderam a pedidos de observação por alterações em exames complementares de diagnóstico, descompensações do estado geral ou de sistemas orgânicos específicos, e ainda intercorrências infeciosas. Relativamente ao período temporal dos pedidos, verificou-se que 32 foram feitos logo à admissão do doente, 29 ainda em tempo pré-operatório e 27 posteriormente.

Apesar de não se contabilizaram neste trabalho, registaram-se 13 casos em que não foi feito pedido de colaboração da Ortopedia por escrito, tendo sido apenas solicitado o apoio de Medicina Interna verbalmente e prestado igualmente o devido apoio.
A maioria dos doentes exigiu visita diária ( $n=52,59 \%$ dos casos) e apresentava outros problemas de relevo além do motivo pelo qual foi feito o pedido de observação ( $n=55,62,5 \%$ dos casos).

Em 17 doentes (19,3\% dos casos) não se registaram comorbilidades importantes, enquanto nos restantes estas estavam presentes em número elevado.

Nos doentes avaliados por Medicina Interna foram diagnosticadas uma variedade de entidades nosológicas, e tratadas adequadamente, sempre a par com o acompanhamento do problema ortopédico. Os principais problemas seguidos estão discriminados na Tabela 1. Um número importante dos doentes ( $n=54,61 \%$ dos casos) estava medicado com antiagregação plaquetária (simples ou dupla) ou hipocoagulação, exigindo a respetiva avaliação e ajuste pré-operatório quando aplicado.

Foi necessária a instituição de antibioterapia em 19 doentes ( $21,5 \%$ dos casos) e foram detetadas infeções nosocomiais em 16 doentes (18,2\% dos casos). Registou-se dor importante em 10 doentes, tendo os restantes 78 sido bem controlados com as medidas analgésicas habitualmente instituídas. 


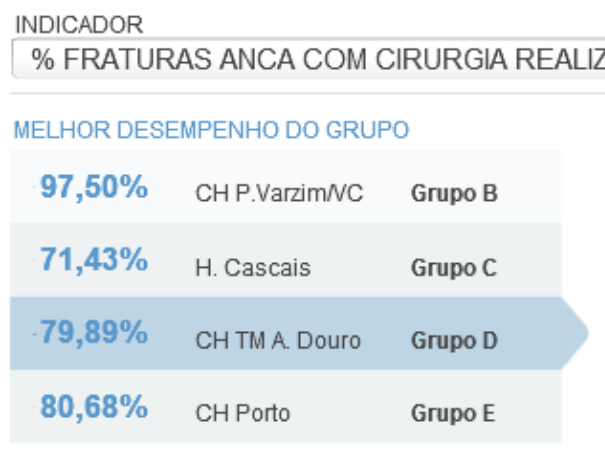

ALINHAMENTO PELO MELHOR DESEMPENHO DO GRUPO

\section{BENCHMARKING GRUPOD}

\% Fraturas da Anca com Cirurgia Realizada nas Primeiras 48 horas (Valores Acumulasos)

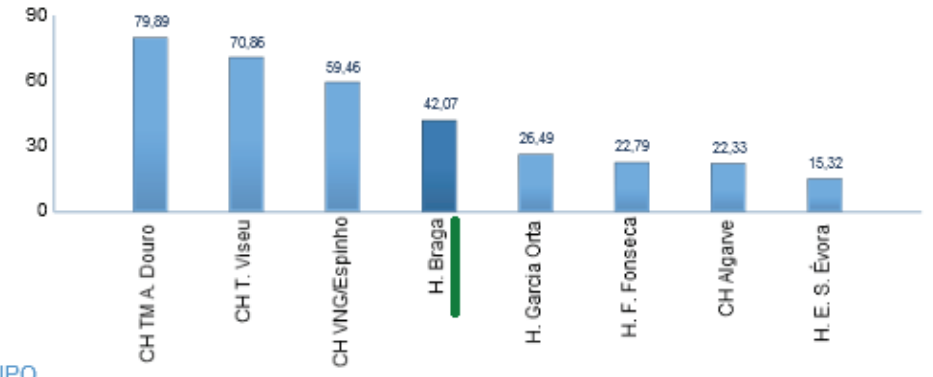

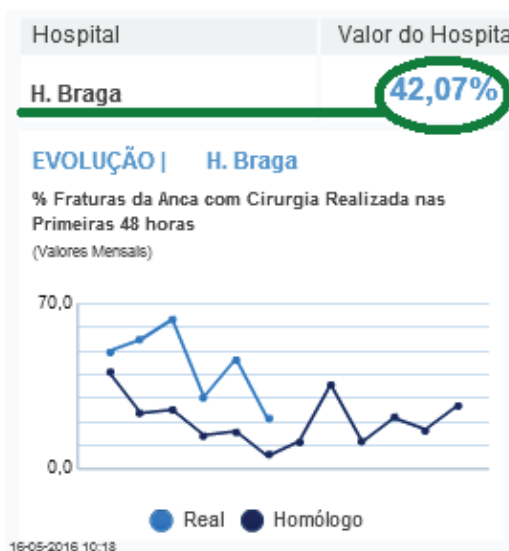

\begin{abstract}
Valor Índice
\end{abstract}
0,527

EVOLUÇÃO। H. Braga

$N^{2}$ Episódios utentes idade sup 65 anos com diag princ $820 \mathrm{com}$ cir realiz nas 1 as $48 \mathrm{H}$ após admissão (Vabres Mensals)

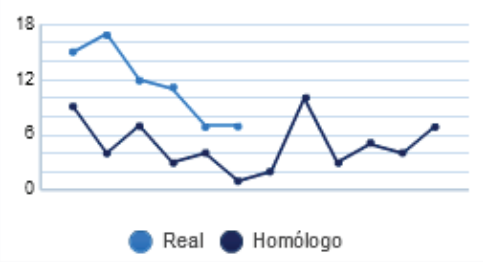

EVOLUÇÃO। H. Braga

$N^{*}$ Episódios utentes idade sup 65 anos com diag princ 820 com cirurgia realizada (Vabres Mensals)

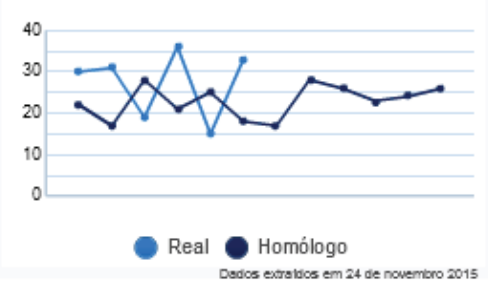

INDICADOR
\begin{tabular}{|lll}
\hline$\%$ FRATURAS ANCA COM CIRURGIA REALIZAD C R & \\
\hline & MELHOR DESEMPENHO DO GRUPO \\
$96,30 \%$ & CH P.VarzimNC & Grupo B \\
$77,50 \%$ & ULS Alto Minho & Grupo C \\
\hline $84,62 \%$ & CH TM A. Douro & Grupo D \\
\hline $86,71 \%$ & CH Porto & Grupo E \\
\hline
\end{tabular}

ALINHAMENTO PELO MELHOR DESEMPENHO DO GRUPO

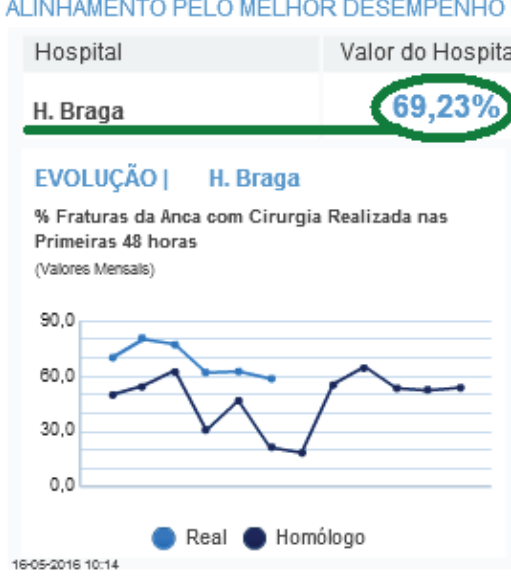

BENCHMARKING GRUPO D

\% Fraturas da Anca com Cirurgia Realizada nas Primeiras $\mathbf{4 8}$ horas

(Valores Acumulaso6)

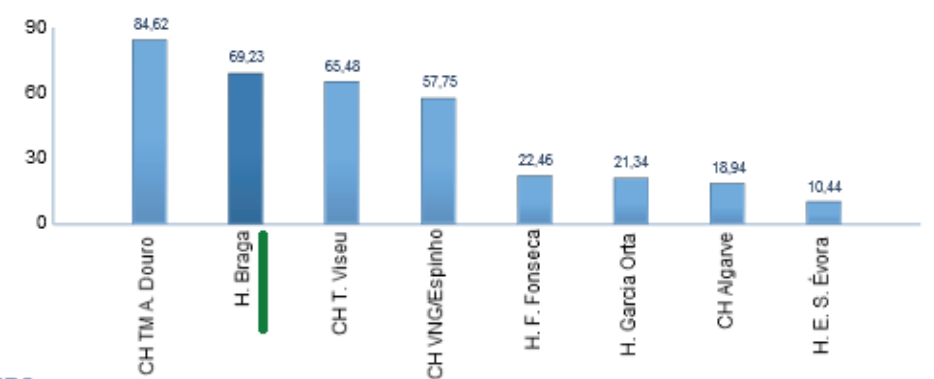

FIGURA 4. Benchmarking de Hospitais - dados disponibilizados pelo SNS e ACSS para o indicador percentagem de fraturas da anca com cirurgia realizada nas primeiras 48 h. (in http://benchmarking.acss.min-saude.pt/benchmarking, acedido em 17/5/2016). 


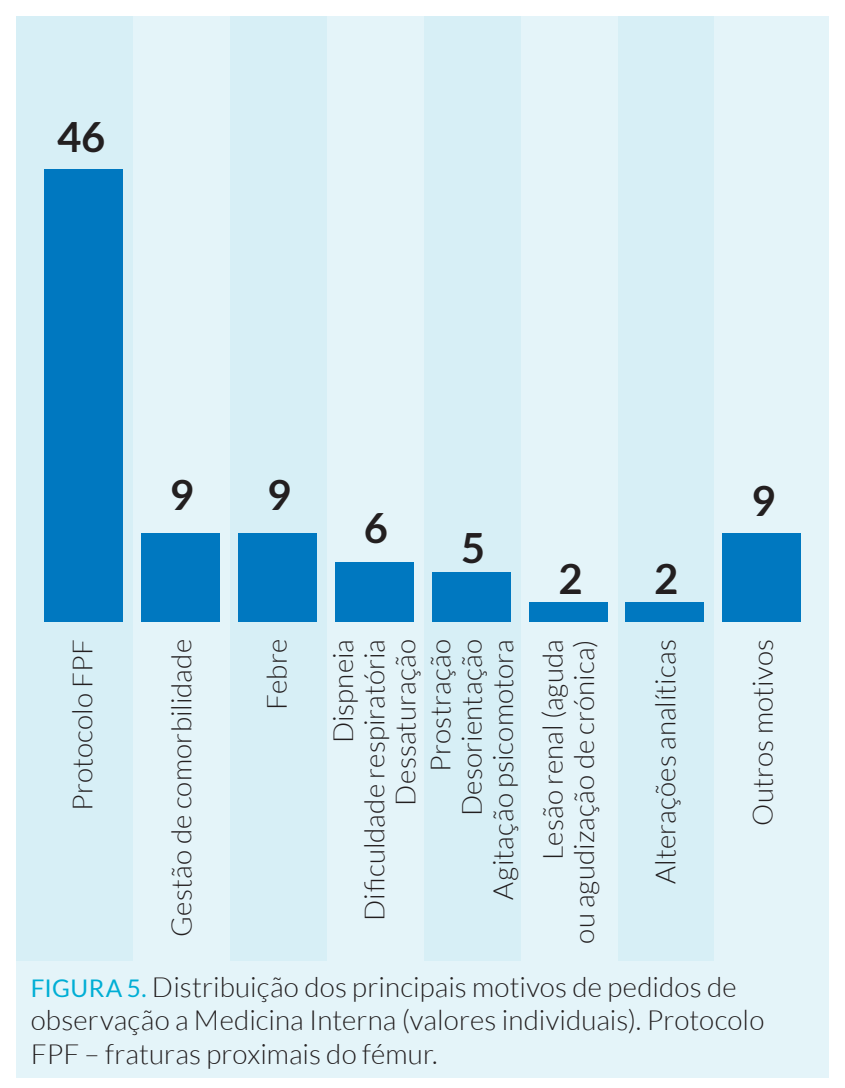

Em 13 doentes foi feito pedido de reobservação, apenas um pelo mesmo motivo (agravamento clínico de celulite), e os restantes por motivos diferentes.

Foram feitos três pedidos de colaboração a outras especialidades por parte da Medicina Interna: um a Otorrinolaringologia por epistáxis abundantes, um a Urologia por hematúria persistente, tendo vindo a diagnosticar-se neoplasia vesical e o doente sido referenciado para a consulta externa dessa especialidade, e um a Cirurgia Geral por icterícia e mal-estar geral, tendo-se feito o diagnóstico de adenocarcinoma das vias biliares; este doente foi transferido para o Serviço de Cirurgia Geral. Não houve registo de nenhuma transferência para o Serviço de Medicina Interna. Apenas em 17 doentes (19,3\% dos casos) não foi necessário nenhum tipo de intervenção por parte da Medicina Interna, correspondendo a situações em cujo pedido foi feito por protocolo FPF ou para gestão de comorbilidades e em que a situação clínica do doente se encontrava estável.

À data de alta, foram orientados para a consulta de Medicina Interna cinco doentes. Registou-se apenas um óbito na amostra analisada (um doente do sexo masculino, de 79 anos, com fratura de vértebra dorsal e bacteriemia por Escherichia coli).

\section{DISCUSSÃO}

O sistema de saúde português tem que se adaptar à alteração progressiva dos padrões demográficos, epidemiológicos e económicos do país. ${ }^{4}$ Cada vez mais se apela à melhoria contínua da qualidade, como um imperativo para gestores, profissionais de saúde e cidadãos; isto implica trabalhar no sentido de cuidados efetivos e seguros, para que a utilização dos recursos seja eficiente, equitativa e atempada.

As fraturas proximais do fémur são lesões traumáticas relativamente frequentes que ocorrem preferencialmente em pessoas de idade avançada, com estrutura óssea fragilizada pela osteoporose. A maior parte das fraturas nesta faixa etária ocorre por quedas da própria altura, sendo mais frequentes no sexo feminino. $\bigcirc$ tratamento deste tipo de lesões é cirúrgico na maioria dos casos, pois a alternativa da tração no membro afetado até à consolidação da fratura está associada a maior tempo de internamento, além de maior probabilidade de complicações como trombose venosa profunda, embolia pulmonar, intercorrências infeciosas e úlceras de decúbito. A opção cirúrgica varia desde a redução cirúrgica e osteossíntese (fixação com implantes que vão manter a fratura alinhada e estável até à sua consolidação) até à artroplastia (substituição por uma prótese) total ou parcial. É necessário ter presente que independentemente do tratamento, e atendendo à população atingida (maioritariamente idosa) são sempre fraturas com considerável mortalidade e morbilidade.,56 Pode-se afirmar, assim, que se o tratamento cirúrgico atempado (idealmente nas primeiras 48 h) é um pilar fundamental do sucesso, existem dois "cofatores" estruturais a um prognóstico favorável: a gestão atenta das comorbilidades e intercorrências do doente pré e pós-cirurgia, e a reabilitação funcional a posteriori. Com o tempo e a familiarização dos colegas de Ortopedia com o protocolo FPF, foi-se verificando um aumento progressivo da sua implementação. Nesta amostra, apenas 14 doentes candidatos a protocolo FPF não foram referenciados como tal. Este trabaIho pretendeu, assim, fazer uma breve abordagem clínica dos doentes ortopédicos com necessidade de apoio de Medicina Interna, não só os que se enquadraram no protocolo FPF, mas todos aqueles que exigiram uma avaliação multidisciplinar. Foi surpreendente verificar a elevada faixa etária dos doentes internados em Ortopedia, embora seja necessário ter em conta que a amostra é relativa aos doentes em que foi feito pedido de colaboração de Medicina Interna, e não a todos os doentes do Serviço de Ortopedia. Foi interessante constatar também que apesar de um elevado número de doentes ser autónomo e estar internado em contexto de situação traumática, na maioria dos casos existiam comorbilidades que exigiam atenção e também na maioria foi necessário apoiar outras situações além do motivo de pedido inicial de observação. Neste seguimento, destaca-se ainda a existência de pedidos de colaboração não apenas numa situação pré-operatória, mas também em contex- 


\section{TABELA 1. Principais entidades patológicas/problemas acompanhados pela Medicina Interna (valores individuais).}

\begin{tabular}{|l|l|}
\hline Sistema cardiovascular & \multicolumn{2}{l|}{} \\
\hline Insuficiência cardíaca descompensada & 9 \\
\hline $\begin{array}{l}\text { Fibrilação auricular com resposta } \\
\text { ventricular rápida }\end{array}$ & 2 \\
\hline Taquicardia sinusal & 1 \\
\hline Sistema respiratório & 1 \\
\hline $\begin{array}{l}\text { Agudização de sintomas de patologia } \\
\text { respiratória crónica (DPOC) }\end{array}$ & 1 \\
\hline Síndrome de hipoventilação-obesidade & 1 \\
\hline Roncopatia & 1 \\
\hline Sistema urinário & 1 \\
\hline Lesão renal aguda (LRA) & 5 \\
\hline Doença renal crónica agudizada & 3 \\
\hline Sistema gastrointestinal & 1 \\
\hline Vómitos & 1 \\
\hline Epigastralgias & 1 \\
\hline Distúrbios iónicos & 1 \\
\hline Hipercaliemia & 1 \\
\hline Hiponatremia & 1 \\
\hline Distúrbios metabólicos & 1 gástrica \\
\hline Sistémica (SRIS) pós-operatória & 1 \\
\hline Diabetes mellitus descompensaliferativa
\end{tabular}

to pós-cirurgia, nomeadamente por analgesia ineficaz, correção de desequilíbrios hidroeletrolíticos e intercorrências infeciosas; de facto, o número de infeções não ortopédicas e a necessidade de instituição de antibiótico foram também em número importante. Todos os dados parecem, por isso, apontar para uma necessária gestão simultânea de problemas relativos a diferentes sistemas orgânicos.

\section{CONCLUSÃO}

O Serviço de Ortopedia do Hospital de Braga é o maior serviço do Hospital no que concerne ao volume de cirurgias efetuadas e a interligação direta e diária com a Medicina Interna, integradora das circunstâncias do doente pretende, por isso, ser uma mais-valia, permitindo otimizar comorbilidades e reduzir taxa de complicações. De facto, registou-se um elevado número de pedidos de observação e os doentes apresentavam, na sua maioria, situações clínicas complexas. Os autores consideram, assim, que articular o cuidado ortopédico com a atenção diária mais holística da Medicina Interna certamente permitirá obter melhores resultados do que uma observação esporádica em contexto de consulta interna.

Os autores estão conscientes que este trabalho representa apenas um primeiro passo. Seria importante elaborar um estudo mais alargado no tempo e comparativo entre o período prévio e o período após colaboração diária da Medicina Interna, nomeadamente no que respeita a avaliação do impacto no tempo de internamento, no grau de morbimortalidade e nos resultados de follow-up dos doentes, nomeadamente numa avaliação 30 dias após a alta. Por enquanto, fica certamente presente a noção real de que é imprescindível um envolvimento multidisciplinar precoce assente nos cuidados da Ortopedia e da Medicina Interna em articulação com a equipa de enfermagem e com outras especialidades eventualmente necessárias para uma otimização pré-operatória dos doentes e para uma melhor continuidade pós-operatória com vista à reabilitação e a melhores outcomes.

CONFLITOS DE INTERESSE: Os autores declaram a inexistência de conflitos de interesse na realização do trabalho.

FONTES DE FINANCIAMENTO: Não existiram fontes externas de financiamento para a realização deste artigo.

CONFIDENCIALIDADE DOS DADOS: Os autores declaram ter seguido os protocolos do seu centro de trabalho acerca da publicação dos dados de doentes.

\section{REFERÊNCIAS}

1. Marshall HW. Some relationships between Ortopedic surgery and Internal Medicine. Boston Med Sur J. 1913;46-8.

2. Barros P, Machado S, Nunes J, Simões A. General Internists at the hospital: cost saving or big spenders? - 6th Congress European Federation of Internal Medicine. 2007.

3. Barros P, Simões J. Portugal Health System review. Europ Obs Health Syst Policies. 2007;9:5.

4. Direção Geral de Saúde. Plano Nacional de Saúde - revisão e extensão a 2020. Lisboa: DGS; 2015

5. Kesmezacar H, Ayhan E, Unlu MC, Seker A, Karaca S. Predictors of mortality in elderly patients with an intertrochanteric or a femoral neck fracture. J Trauma. 2010;68:153-8.

6. Schnell S, Friedman S, Mendelson D, Bingham K, Kates S. The I-Year mortality of patients treated in a hip fracture program for elders. Geriatr Orthop Surg Rehabil. 2010;1:6-14. 